\title{
Body talk in the digital age: A controlled evaluation of a classroom-based intervention to reduce appearance commentary and improve body image
}

Health Psychology Open January-June 202 I: I-12 (c) The Author(s) 2021 Article reuse guidelines: sagepub.com/journals-permissions DOI: 10.1177/20551029211018920 journals.sagepub.com/home/hpo

@SAGE

\author{
Beth T Bell' 1 , Caitlin Taylor', Danielle L. Paddock', \\ Adam Bates 2 iD and Samuel T Orange ${ }^{3}$
}

\begin{abstract}
This research evaluates the efficacy of a classroom-based intervention - Body Talk in the Digital Age (BTIDA) - in reducing adolescents' appearance commentary and improving body image. British adolescents $(N=3 \mid 4$; Age Range $=12-14)$ were cluster randomised to intervention or waiting-list control groups. Measures of appearance commentary, appearance ideal internalisation, self-objectification and body satisfaction were completed at baseline (TI), then one-week (T2) and eight-week (T3) post-intervention. Multi-level modelling showed girls who received BTIDA reported less appearance commentary engagement and thin ideal internalisation at T2 and T3, than the control, supporting the partial efficacy of BTIDA for girls. No intervention effects were found among boys.
\end{abstract}

\section{Keywords}

adolescents, appearance commentary, body image, body talk, intervention, social media

Adolescents engage in, and are exposed to, a variety of appearance-related comments on a daily basis, in both online and offline settings (Bell, 2019; Jones and Crawford, 2006; Puhl et al., 2017). These interactions can take many forms, encompassing both positive ('You look amazing') and negative comments ('She looks way too fat in that dress') about their own bodies ('I wish my thighs were thinner') and those of others ('He is ripped'), and can be both positively or negatively intended. However, appearance-related interactions - both positive and negative - have been linked to negative body image (Jones, 2004; Jones et al., 2014; Lawler and Nixon, 2011; Puhl et al., 2017). Challenging appearance-focused interactions with peers in offline settings is a feature of existing effective classroom-based interventions that seek to improve adolescents' body image and reduce eating disorder risk, but their effectiveness in reducing these interactions is mixed (e.g. Dunstan et al., 2017; McLean et al., 2017; Richardson and Paxton, 2010). The present study aims to evaluate the effectiveness of a brief classroombased intervention - Body Talk in the Digital Age (BTIDA) - in reducing adolescents' on- and off- line appearance commentary and improving body image.

\section{Negative body image and adolescence: $A$ sociocultural perspective}

Negative body image refers to the experience of negative thoughts and feelings towards one's own body, incorporating the experience of body dissatisfaction and shame, as well as overinflated beliefs about the importance of physical appearance (Grogan, 2016; Holmqvist Gattario and Frisén, 2019). It has been linked concurrently and prospectively to a wide range of negative outcomes, including low self-esteem, depressed mood and disordered eating (Cruz-Sáez et al., 2018; Neumark-Sztainer et al., 2006), and is both a risk factor for, and symptomatic of, clinical eating disorders (Rohde et al., 2015; Smolak and Levine,

\footnotetext{
'York St John University, UK

${ }^{2}$ Sheffield Hallam University, UK

${ }^{3}$ Newcastle University, UK
}

Corresponding author:

Beth T Bell, School of Education, Languages and Psychology, York St John University, Lord Mayor's Walk, York YO3I 7EX, UK.

Email: b.bell@yorksj.ac.uk 
2015). Negative body image is highly prevalent among adolescents: a recent survey found $52 \%$ of $11-16$-year olds in the UK worry about their appearance and 30\% isolate themselves because of this (Be Real, 2017).

Sociocultural theories, including the tripartite model (Thompson et al., 1999) and objectification theory (Fredrickson and Roberts, 1997), emphasise the role of social and cultural factors, including peers, parents and media, in the aetiology of negative body image. According to these theories, adolescents are repeatedly exposed to messages emphasising the importance of physical attractiveness (Fredrickson and Roberts, 1997), which is defined in terms of culturally prescribed, unrealistic and artificial body and beauty ideals (Thompson et al., 1999). Over time, adolescents internalise these messages, eventually leading to the experience of body dissatisfaction as they perceive themselves as failing to live up to the unrealistic standards of physical attractiveness set by society (e.g. Dakanalis et al., 2015; Knauss et al., 2008).

Understood within the lens of sociocultural theories, appearance commentary serves to reproduce and reinforce problematic sociocultural messages surrounding appearance in both direct (e.g. 'You look amazing - have you lost weight?') and indirect ways (e.g. 'I've eaten so much, I feel like a fatty'). A diverse body of research supports the link between engaging in, being a recipient of, and being exposed to, appearance commentary in everyday life and body image disturbance among adolescents and young adults (Chen and Jackson, 2012; Jackson and Chen, 2011; Jones, 2004; Jones and Crawford, 2006; Jones et al., 2014; Lawler and Nixon, 2011; Nichter, 2000; Shannon and Mills, 2015; Tiggemann and Barbato, 2018; Trekels et al., 2018). Much of this research has focused on girls, who typically report higher levels of appearance commentary engagement than their male counterparts (e.g., Jones and Crawford 2006; Trekels et al., 2018), as well as higher levels of appearance ideal internalisation and body dissatisfaction (Dakanalis et al., 2015; Karazsia et al., 2017). That said, research suggests that boys do still engage in appearance-related interactions, particularly appearance-related teasing, and this engagement has similarly been linked to body dissatisfaction (Chen and Jackson, 2012; Jackson and Chen, 2011; Jones, 2004; Jones and Crawford, 2006).

In contemporary society, social media platforms are widely used to facilitate adolescents' interpersonal interactions (boyd, 2014; Livingstone, 2019). Social media platforms that centre interactions around user-generated images, such as Instagram and Snapchat, are particularly popular among adolescents (Ofcom, 2020). Many of the images shared through these sites (e.g. selfies) are typically edited to conform to unrealistic body and beauty ideals (e.g. Deighton-Smith and Bell, 2018; Döring et al., 2016), which adolescents report responding to in appearance-focused ways, e.g., complimenting appearance (Bell, 2019; Chua and Chang, 2016). In addition, some adolescents describe posting self-images with the intention of eliciting positive appearance feedback from others (Bell, 2019; Chua and Chang, 2016). Thus, social media platform design may further encourage appearance-related interactions among adolescents.

\section{Interventions to improve adolescent body image}

Multiple interventions exist for improving adolescent body image and reducing eating disorder risk (Chua et al., 2020; Lewis-Smith et al., 2019; Yager et al., 2013). These interventions are typically delivered in schools, since the classroom allows for inclusive and cost-effective delivery (Chua et al., 2020; Yager et al., 2013). Effective interventions typically aim to foster cognitive dissonance and/or critical literacy through the verbalising of critiques of sociocultural appearance pressures and the rehearsal of strategies to protect the self from these pressures (Lewis-Smith et al., 2019). In controlled trials, interventions utilising these approaches have demonstrated effectiveness in improving some, but not all, measured dimensions of body image, body image risk (e.g. internalisation of appearance ideals) and disordered eating among adolescents (Chua et al., 2020; LewisSmith et al., 2019; Yager et al., 2013).

Challenging appearance commentary is a common feature of many classroom-based interventions (Bird et al. 2013; Dunstan et al., 2017; Halliwell et al., 2015; McLean et al. 2017; Richardson and Paxton, 2010; Wilksch et al., 2015). Most notably, Happy Being Me (HBM) was found to reduce Australian girls' (aged 1114 years old) body dissatisfaction and thin ideal internalisation (Dunstan et al., 2017; Richardson and Paxton, 2010) in controlled trials. Subsequent adaptations of HBM have also shown some efficacy, improving some, but not all, measured aspects of body image (Bird et al., 2013; Wilksch et al., 2015). Most recently, SoMe was developed as an adaptation of $H B M$ to focus on the specific challenges posed by social media environment (McLean et al., 2017). It was found to effectively improve some aspects of adolescent girls' body image and media literacy post-intervention (McLean et al., 2017). However, the effectiveness of these programmes in reducing appearance commentary is more mixed. Only Richardson and Paxton (2010) reported positive effects of the intervention on appearance commentary that were sustained at follow-up. Bird et al. (2013) found an effect for girls that was not sustained, whereas others found no impact (Dunstan et al., 2017; McLean et al., 2017) or did not measure this (Wilksch et al., 2015).

Given that appearance commentary is behavioural, it may be that the effectiveness of these interventions (i.e. those that use cognitive dissonance and critical literacy to 
challenge appearance commentary) may be improved by incorporating change techniques explicitly focused on behaviour. One such technique, implementation intentions, involves formulating specific 'If-Then' plans that specify where, when and how to act in a given situation ('If I encounter X, then I will perform Y'), in order to facilitate behaviour change (Gollwitzer and Sheeran, 2006; Wieber et al., 2015). Implementation intentions has demonstrated efficacy in reducing problem health behaviour in a variety of domains (see Wieber et al. 2015 for review), but not in relation to appearance commentary. Adding an implementations intention activity towards the end of a classroom-based body image intervention may support adolescents to reduce appearance commentary outside the classroom, by providing them with a specific behavioural plan that is consistent with the critical views that they have articulated within the classroom. Research has also suggested that the effectiveness of implementations intentions in creating behaviour change can be enhanced when used in conjunction with a self-affirmation task (e.g. Harris et al., 2014). Self-affirmation involves encouraging focus on positive aspects of the self, for example, important values, attributes or past behaviours, in order to reduce defensiveness to counterattitudinal messages, making behaviour change more likely (Epton et al., 2015). It is typically used at the start of interventions to increase participants' receptiveness to behaviour change messages (Epton et al., 2015). Thus, we aim to bookend our cognitive dissonance and critical literacy intervention, with a self-affirmation activity at the start and implementation intentions activity at the end, to enhance the efficacy of the intervention in reducing appearance commentary.

A second issue with existing interventions is duration. Reviews and meta-analyses have consistently shown that multiple session interventions are most effective in improving adolescents' body image (Chua et al., 2020; Lewis-Smith et al., 2019; Yager et al., 2013). However, current educational policies have marginalised aspects of health education and allow little school-time for their delivery (Tancred et al., 2017), meaning there may be practical reasons for preferring single-session interventions. Promisingly, there is some evidence that single-session classroom interventions using cognitive dissonance as their underpinning mechanism can be effective in reducing body image disturbance in the short term (Diedrichs et al., 2015; Halliwell et al., 2015) but not in the long-term (Diedrichs et al., 2015). By adding activities based on implementation intentions and self-affirmation to our intervention, which have demonstrated effectiveness in reducing problem behaviour in singlesession interventions (Epton et al, 2015; Wieber et al., 2015), we hope to enhance the ability of our single session intervention to create more lasting reductions in appearance commentary and improvements in body image.

\section{The present study: Body talk in the digital age}

The present study aims to evaluate the effectiveness of Body Talk in the Digital Age, a single-session classroombased intervention that aims to reduce adolescents' engagement in appearance commentary in both offline and online settings and improve body image. It is predicted that adolescents who participate in the intervention will report lower levels of appearance commentary engagement, selfobjectification, appearance (thin and athletic) ideal internalisation and higher levels of body satisfaction post-intervention and at 8-week follow-up, than participants assigned to a waiting list control. Furthermore, based on existing research demonstrating that appearance conversations and body image issues are more prevalent among girls (e.g. Dakanalis et al., 2015; Jones and Crawford 2006; Karazsia et al., 2017; Trekels et al., 2018) and also that classroom-based interventions are typically more effective for girls (e.g. Yager et al., 2013), we predict that intervention effects will be stronger among girls.

\section{Method}

\section{Participants}

Participants were recruited from the same co-educational high school in Northern England, representing the entirety of two different year groups $(N=314$; Year $8, n=153$, Age $M=12.62, \mathrm{SD}=0.49$, Range $=12-13$, Female $n=71$; Year $10, n=161$, Age $M=14.64$, Range $=14-15, \mathrm{SD}=0.48$; Female $n=76$ ). Aside from age and gender, no additional demographic information was collected at the individual level. However, publicly available information about the school shows attending students are mostly White British, with a lower than national average proportion living in low SES households. Adolescents participated in the intervention within their normal class group. To assign groups to condition (experimental or waiting list control), classes within each year group were paired according to academic ability by a teacher, then one class from each pair was randomly allocated to each condition. Participant recruitment and allocation are outlined in Figure 1.

\section{Intervention}

The BTIDA intervention involved one lesson lasting sixty minutes. The content of the session was focused on identifying and critiquing appearance commentary, including in social media interactions. As per previous effective interventions, the main body of the intervention was delivered through interactive activities aimed at building cognitive dissonance and critical literacy skills (Lewis-Smith et al., 2019). In addition, adolescents engaged in a self-affirmation activity at the start of the session to help overcome any potential resistance (Epton et al., 2015). They also engaged in an implementation intentions exercise at the end of the 


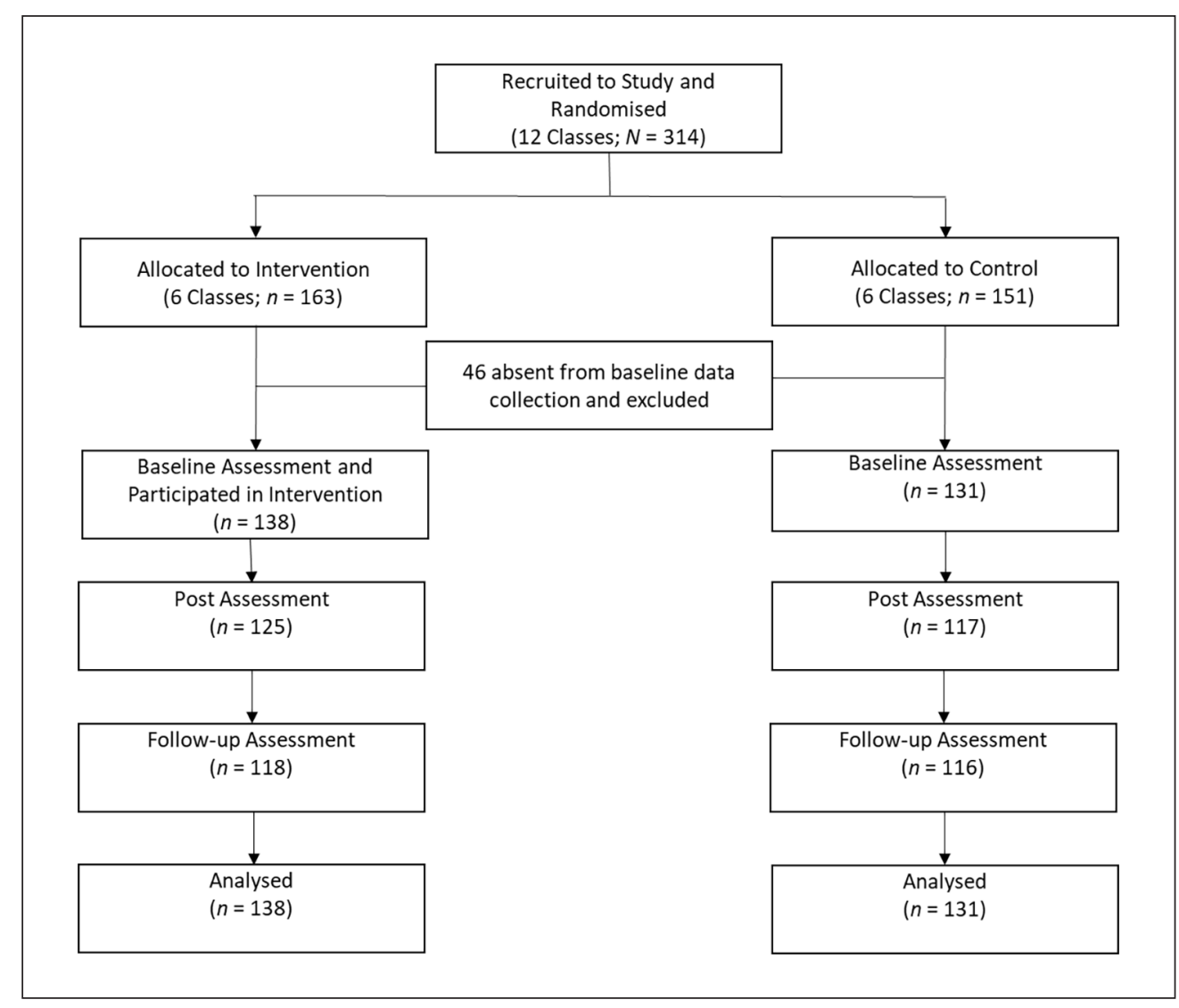

Figure I. Participant recruitment, allocation, retention and flow.

session, formulating specific 'If-Then' plans to reduce their engagement in appearance commentary (Gollwitzer and Sheeran, 2006; Wieber et al., 2015). Content and activities are shown in Table 1.

BTIDA was designed by the first author in collaboration with an experienced teacher (Author 4), with over 20 years of experience delivering health education in schools. It was initially developed as a suggested lesson plan, given to teachers as part of a professional development course about body image, and had received very favourable reviews in followup questionnaires $(n=10)$, in terms of interest/enjoyment $(M=4.90, \quad \mathrm{SD}=0.32, \quad$ Scale $=1-5)$ and impact/learning $(M=4.80, \mathrm{SD}=0.42$, Scale $=1-5)$. Prior to the current evaluation, the session was piloted with 120 adolescent boy and girls (aged 13-14years old). In survey data collected as part of the pilot, the session received favourable ratings from adolescents on a five-point scale, in terms of interest $(M=3.88$; $\mathrm{SD}=0.96)$, learning $(M=3.85 ; \mathrm{SD}=0.92)$ and enjoyment $(M=4.06 ; \mathrm{SD}=1.00)$. Furthermore, qualitative feedback suggested that session had been effective in encouraging them to reflect critically on their everyday use of appearance commentary in online settings (e.g. 'I had not realised how much my Instagram comments might affect me and my friends until now'). Following the pilot delivery of the session, some small modifications to delivery were made (e.g. inclusion of a short activity that introduced unrealistic appearance ideals to help contextualise latter discussions). Pilot data is available on the Open Science Framework (https://osf.io/45nhm/?view_only $=$ fda780e7c80a489397dbafb466332ab9).

\section{Measures}

Demographic information was collected prior to study commencement, at the same time as consent was acquired. Additionally, measures of the following constructs were completed at three time points (pre- intervention, postintervention and 8-week follow-up):

Appearance commentary. A short novel measure was created to assess participants' engagement in appearance commentary, based on a measure of fat talk by Salk and Engeln-Maddox (2011). Participants were provided with a definition of appearance commentary, which was referred to as body talk for the purposes of the intervention and defined as 'the comments we make about our own bodies, or someone else's body, including any appearance-related comparisons we might make. Body talk can be positive or negative' along with examples, including 'Have you lost weight? You look amazing', 'He's ripped', 'Does my bum look big in this?', and 'I wish my arms were bigger". To 
Table I. Overview of body talk in the digital age intervention activities.

\begin{tabular}{|c|c|}
\hline Activity & Description \\
\hline Self-affirmation & $\begin{array}{l}\text { Participants identify one thing that is important to them and explain why it is important in their work- } \\
\text { book. [5 minutes] }\end{array}$ \\
\hline $\begin{array}{l}\text { Introduction to } \\
\text { 'body perfect' } \\
\text { ideals }\end{array}$ & $\begin{array}{l}\text { In groups, participants draw the sociocultural "body perfect" appearance ideal. Groups then share this with } \\
\text { the rest of the class to highlight similarities. The idea of a perfect body is then critiqued as something that is } \\
\text { unrealistic and impossible to attain for the majority of people, and the class then discuss where these ideas } \\
\text { about the body perfect originate from (including peers, media, social media, cartoons etc.). [ } 10 \text { minutes] }\end{array}$ \\
\hline $\begin{array}{l}\text { Introduction to } \\
\text { body talk }\end{array}$ & $\begin{array}{l}\text { Participants are introduced to the concept of 'body talk' (i.e. appearance commentary) by the facilitator } \\
\text { using examples, who explains how and why body talk reinforces body ideals, and how it can make people } \\
\text { feel bad about their body. [ } 5 \text { minutes] }\end{array}$ \\
\hline $\begin{array}{l}\text { Body talk is } \\
\text { everywhere }\end{array}$ & $\begin{array}{l}\text { Participants are provided with recent magazines and asked to find an example of appearance commentary. } \\
\text { They cut and paste the example in the worksheet and then think of something non-appearance related } \\
\text { that could have been written instead. [ } 10 \text { minutes] }\end{array}$ \\
\hline $\begin{array}{l}\text { Body talk on } \\
\text { social media }\end{array}$ & $\begin{array}{l}\text { Participants discuss examples of body talk on social media. Facilitator describes how aspects of the social } \\
\text { media environment encourage body talk (e.g., being invited to like or comment on images, and trends } \\
\text { such as \#like4rate that invite other users to comment on their appearance in images) and participants are } \\
\text { invited to discuss/share how this makes them and others feel. [ } 10 \text { minutes] }\end{array}$ \\
\hline $\begin{array}{l}\text { What can we } \\
\text { do about it } \\
\text { (implementations } \\
\text { intention)? }\end{array}$ & $\begin{array}{l}\text { Participants are provided with three scenarios where body talk may occur in online settings (including } \\
\text { [i] friend posting a selfie, [ii] viewing and receiving a negative appearance comment, [iii] weight-loss } \\
\text { discussion in a group chat), and instructed to discuss how they might respond to each scenario in } \\
\text { a way that would reduce body talk. They then write their preferred action for each scenario (as an } \\
\text { implementation intention) in their workbook and make a pledge to try and reduce online and offline body } \\
\text { talk by following the behavioural plans they have created. [ } 15 \text { minutes] }\end{array}$ \\
\hline
\end{tabular}

assess engagement in appearance commentary, participants were asked to rate on a five-point scale ( $1=$ never, $5=$ always), how often they 'engage in body talk in everyday conversation' and, separately, 'on social media'. Both items were correlated at each timepoint $(r=>0.51)$, consistent with research that has conceptualised online interactions as an extension of offline interactions, especially in nonymous social media environments (e.g. Bell, 2019; boyd, 2014; Yau and Reich, 2018). Thus, an overall appearance commentary engagement score was created based on the mean of these two items. Furthermore, and though not part of our formal hypotheses, we also assessed adolescents' exposure to other people engaging in appearance commentary, as a proxy of their awareness of appearance interactions in the sociocultural environment. Participants were also asked to rate how often they 'encounter or hear other people engaging in body talk in everyday conversation' and 'encounter or see other people engaging in body talk on social media' on the same 1-5 scale. Again, these two items were highly correlated with one another $(r=>0.51)$, indicating internal reliability.

To ensure face validity of the present scale, the definition of body talk used in this study was presented to adolescents $(n=120)$ at the start of the pilot work described previously. In small groups, adolescents provided verbal feedback on the definition, by responding to questions from the research team (e.g. 'describe in your own words what body talk is?'). In this feedback, some adolescents suggested the inclusion of examples of body talk to help illustrate the concept and provided some suggestions for this.
The examples provided to participants in the present study were taken verbatim from adolescents' suggestions.

Appearance ideal internalisation. The extent to which adolescents had internalised societal appearance ideals as personal goals was assessed using the thinness and athletic internalisation subscales of the Sociocultural Attitudes Towards Appearance Questionnaire (SATAQ-4; Schaefer et al., 2015). Each subscale comprises five items (e.g. 'I want my body to look very lean' and 'I think a lot about looking muscular') that participants rate on a five point scale $(1=$ Definitely disagree, 5=Definitely agree $)$. Mean scores were calculated for each subscale independently to create separate scores for thin ideal and athletic ideal internalisation, with higher scores reflecting higher endorsement. The internalisation subscales of the SATAQ-4 have shown good construct validity and reliability when used with adolescent and young adult samples across cultures (Frisén and Berne, 2020; Schaefer et al., 2015; Yamamiya et al., 2016; 2019). Both subscales demonstrated good internal reliability across all time points in the present study (Time $1 \omega=0.80,95 \% \mathrm{CI}=0.75-0.88$; Time $2 \omega=0.86$, 95\% CI $=0.83-0.88$; Time $3 \omega=0.87,95 \% \mathrm{CI}=0.84-0.91$; Time $1 \omega=0.83,95 \%$ CI $=0.80-0.90$; Time $2 \omega=0.81,95 \%$ $\mathrm{CI}=0.80-0.89$; Time $3 \omega=0.88,95 \% \mathrm{CI}=0.73-0.90)$.

Body satisfaction. The Body Satisfaction Scale (BSS; Bird et al., 2013) was used to assess how adolescents feel about their body. The scale comprises five items (e.g. Do you feel satisfied with your appearance?), which participants 
respond to on a five-point scale ( $1=$ never, $5=$ very often $)$. Items are summed to calculate an overall score of body satisfaction, ranging from 1 to 25 , with higher scores indicating high body satisfaction. By focusing on body satisfaction rather than dissatisfaction through its positively worded questions, the BSS is particularly appropriate for use with younger samples. It has shown good validity and reliability in past intervention research (Bird et al., 2013). In the present study, good internal reliability was observed across time points (Time $1 \omega=0.89,95 \% \mathrm{CI}=0.86-0.91$; Time $2 \omega=0.86,95 \% \mathrm{CI}=0.83-0.89$; Time $3 \omega=0.87$, $95 \% \mathrm{CI}=0.83-0.90)$.

Self-objectification. The Self-Objectification Questionnaire (Noll and Fredrickson, 1998) requires participants to rank a selection of 10 body attributes according to how important they are to their self-concept $(1=$ Not at all important to me, $10=$ Very important to me). Five of the attributes are appearance-based (e.g. sex appeal and physical attractiveness) and five are competence-based (e.g. health and stamina). Scores are calculated by subtracting the sum of the competence attributes from the sum of the appearance attributes (Range $=-25$ to 25). High scores reflect a greater emphasis on the importance of appearance-based physical attributes over competency-based attributes, indicating high levels of self-objectification. The measure has demonstrated validity and reliability in young female samples (Noll and Fredrickson, 1998), and shows the expected pattern of correlation with body image related constructs (indicative of construct validity) in male and female adolescents across a variety of Western cultures (Slater and Tiggemann, 2011; Vandenbosch and Eggermont, 2014).

\section{Procedure}

A three-tier consent process was adopted, wherein informed consent was first gained from the school head-teacher, then opt-out consent was obtained from parents/guardians, and finally opt-in consent was obtained from students on the day of baseline testing. No eligible student chose not to take part in the study. After providing consent at baseline (Time 1) participants provided demographic information and completed measures of appearance commentary, internalisation of appearance messages and body satisfaction. During the following week, participants in the experimental condition received the sixty-minute intervention, which was delivered to each individual class by two trained research assistants (one male and one female) instead of their timetabled religious education lesson. The research assistants had received 4 hours of training in intervention delivery, as well as experience in delivering the intervention to adolescents from the pilot work. One week after the intervention (Time 2), participants completed a second questionnaire containing the same measures as the first. Eight weeks after the intervention, all participants completed the same questionnaire again (Time 3). The waiting list control condition received the intervention during the following week. All questionnaires were paperbased and completed in classrooms in silence. Measures were not counterbalanced within the questionnaires.

At the end of the study, all participants were provided with a short paper-based debrief reminding them of the purpose of the study that also contained details of information services and support organisations. The school also received a short report detailing the study findings. No participation incentives were provided. The study adhered to the British Psychological Society ethical guidelines and received approval from York St. John University ethics committee. Intervention delivery and data collection took place between May and July 2019. The study was not pre-registered, however all study materials (pilot work, intervention materials, data, and analysis code) are available on the Open Science Framework see: https://osf.io/45nhm/?view only=fda780e7c80a489397dbafb466332ab9.

\section{Statistical analysis}

Data were analysed in R version 4.0.0 (R Foundation for Statistical Computing, Vienna, Austria). Descriptive statistics were used to characterise participants at baseline. Between-group differences in outcomes at post-intervention and follow-up were assessed with a multi-level linear model to account for the hierarchical nature of the data (repeated measures nested within participants). Of the recruited participants, 269 were in school on the day of baseline testing and so comprise the sample on which our analyses are based (see Figure 1 for details of participant recruitment, retention and flow). All attrition was due to student absences from school on the day of testing. Under the assumption of missing at random, missing data were handled within the model using the maximum likelihood estimation method. This method has been shown to be superior to multiple imputation for handling missing data in multilevel linear models (Allison, 2012; Shin et al., 2017).

A random intercept-only model was initially fitted for each outcome, allowing intercepts to vary across participants. If adding a random slope to the model did not significantly improve the model fit (assessed via the likelihood-ratio test), it was removed, and the model was re-run with a random intercept only. Fixed effects entered into the model were: time (coded as: baseline $=0$, post-intervention $=1$, follow-up $=2$ ), group $($ control $=0$, experimental $=1)$, gender $\quad($ boys $=0$, girls $=1$ ), the two-way interaction between group and time, and the three-way interaction between gender, group and time. School year was also included in the model as a covariate. All group $\mathrm{x}$ time interaction effects were followed up with a priori planned pairwise comparisons between-groups at each post-intervention time-point. Gender $\times$ group $\times$ time interactions were further explored by conducting separate pairwise comparisons for girls and boys. The $p$-values and 
Table 2. Baseline characteristics of sample according to condition and gender.

\begin{tabular}{|c|c|c|c|c|c|c|c|c|c|c|c|c|}
\hline & \multicolumn{4}{|c|}{ Intervention } & \multicolumn{4}{|c|}{ Control } & \multicolumn{4}{|c|}{ Overall } \\
\hline & \multicolumn{2}{|c|}{ Girls $n=66$} & \multicolumn{2}{|c|}{ Boys $n=72$} & \multicolumn{2}{|c|}{ Girls $n=62$} & \multicolumn{2}{|c|}{ Boys $n=69$} & \multicolumn{2}{|c|}{ Girls $n=128$} & \multicolumn{2}{|c|}{ Boys $n=|4|$} \\
\hline & $M$ & $S D$ & $M$ & $S D$ & $M$ & $S D$ & $M$ & $S D$ & $M$ & $S D$ & $M$ & $S D$ \\
\hline Age & 13.62 & 1.05 & 13.72 & 1.10 & 13.77 & 1.11 & 13.45 & 1.21 & 13.70 & 1.08 & 13.59 & 1.16 \\
\hline AC-Engage & 2.59 & 0.98 & 1.84 & 0.86 & 2.57 & 0.87 & 1.96 & 0.86 & 2.58 & 0.93 & 1.90 & 0.86 \\
\hline AC-Exposure & 2.98 & 0.89 & 2.65 & 1.06 & 3.04 & 0.96 & 2.85 & 1.05 & 3.01 & 0.92 & 2.75 & 1.06 \\
\hline TII & 2.96 & 0.88 & 2.50 & 0.83 & 2.80 & 0.87 & 2.37 & 0.82 & 2.88 & 0.88 & 2.43 & 0.83 \\
\hline All & 2.37 & 0.96 & 2.69 & 1.03 & 2.39 & 0.95 & 2.59 & 0.97 & 2.38 & 0.95 & 2.64 & 1.00 \\
\hline SO & -4.42 & 11.98 & -|| $.3 \mid$ & 11.45 & -5.61 & 11.68 & -11.23 & 10.95 & -5.00 & $\mid 1.81$ & -11.27 & 11.17 \\
\hline BS & 15.25 & 4.08 & 17.54 & 4.18 & 15.48 & 4.58 & 18.17 & 4.14 & 15.36 & 4.32 & 17.85 & 4.16 \\
\hline
\end{tabular}

AC-Engage: appearance commentary engagement; AC-Exposure: appearance commentary exposure; TII: thin ideal internalisation; All: athletic ideal internalisation; SO: self-objectification, BS: body satisfaction.

95\% confidence intervals (CIs) from pairwise comparisons were adjusted with a Bonferroni correction to reduce the family-wise error rate (i.e. by multiplying the $p$-value by 2 ). Model assumptions (e.g. normality, outliers) were verified by visual inspection of residuals via histograms, boxplots and Q-Q plots. Statistical significance was set at $p<0.05$. We also calculated Cohen's $d$ as a measure of standardised effect size between-groups using the formula: ([mean change intervention group - mean change control group]/pooled SD of change scores). By convention, standardised effect sizes were rated as trivial $(<0.2)$, small $(0.2-0.49)$, medium $(0.50-0.79)$, or large $(\geqslant 0.8)$ (Cohen, 1988).

\section{Results}

\section{Participant baseline characteristics}

Demographic characteristics of the final sample are shown in Table 2. A series of $2 \times 2$ ANOVAs were run to assess whether there were any differences between participants at baseline according to gender (male vs female) and condition (intervention vs control). Though there were no significant baseline differences between boys and girls in terms of age $(F(1,265)=0.67, p>0.05)$, they significantly differed across all other study variables. As Table 2 shows, girls reported significantly higher levels of appearance commentary engagement and exposure, thin appearance ideal internalisation, and self-objectification, but lower muscular ideal internalisation and body satisfaction $(F(1,265)=4.59-38.82$, all $p<0.05)$. No significant differences were found between conditions for age or any outcome variable $(F(1,265)=0.11-$ 2.03 , all $p>0.05)$, and nor were any group $\mathrm{X}$ gender interaction effects found $(F(1,265)=0.03-2.43$, all $p>0.05)$.

\section{Intervention effects}

As Table 3 shows, there were significant interactions effects between group and time for appearance commentary engagement $(p<0.001)$, self-objectification $(p=0.0495)$, and thin ideal internalisation $(p<0.001)$. Subsequent pairwise comparisons revealed that the intervention significantly reduced appearance commentary engagement and thin ideal internalisation post-intervention and at 8-week follow-up, compared to the control group. Whereas effect sizes for appearance commentary engagement were small post-intervention $(d=0.25)$ and at follow-up $(d=0.27)$, they were large for thin ideal internalisation post-intervention $(d=0.82)$ and medium at follow-up $(d=0.72)$. The intervention group also significantly reported increased appearance commentary exposure at 8 -week follow-up compared with the control group, but not immediately post intervention. This effect was medium-sized $(d=-0.54)$.

\section{Moderating effects of gender}

There were significant group $\mathrm{x}$ time $\mathrm{x}$ gender interaction effects for appearance commentary exposure $(p<0.001)$, self-objectification $(p=0.04)$, and thin ideal internalisation $(p<0.001)$. Subsequent pairwise comparisons demonstrated different effects of the intervention for girls and boys (Table 4). For girls, the intervention significantly reduced appearance commentary engagement and thin ideal internalisation at post-intervention and 8-week follow-up, compared with the control group. In contrast, there was no evidence for an effect of the intervention among boys. Furthermore, there was a significant between-group difference in appearance commentary exposure. Girls in the intervention group reported increased appearance commentary at 8-week follow-up compared to the control, whereas there was no significant difference between groups among boys. The majority of these effect sizes were large (thin ideal internalisation post- $d=1.49$, follow-up $d=1.21$; appearance commentary engagement post- $d=0.80$ ), with the exception of appearance commentary engagement and exposure at follow-up ( $d=0.72, d=-0.72$, respectively), where medium-sized effects were found. 

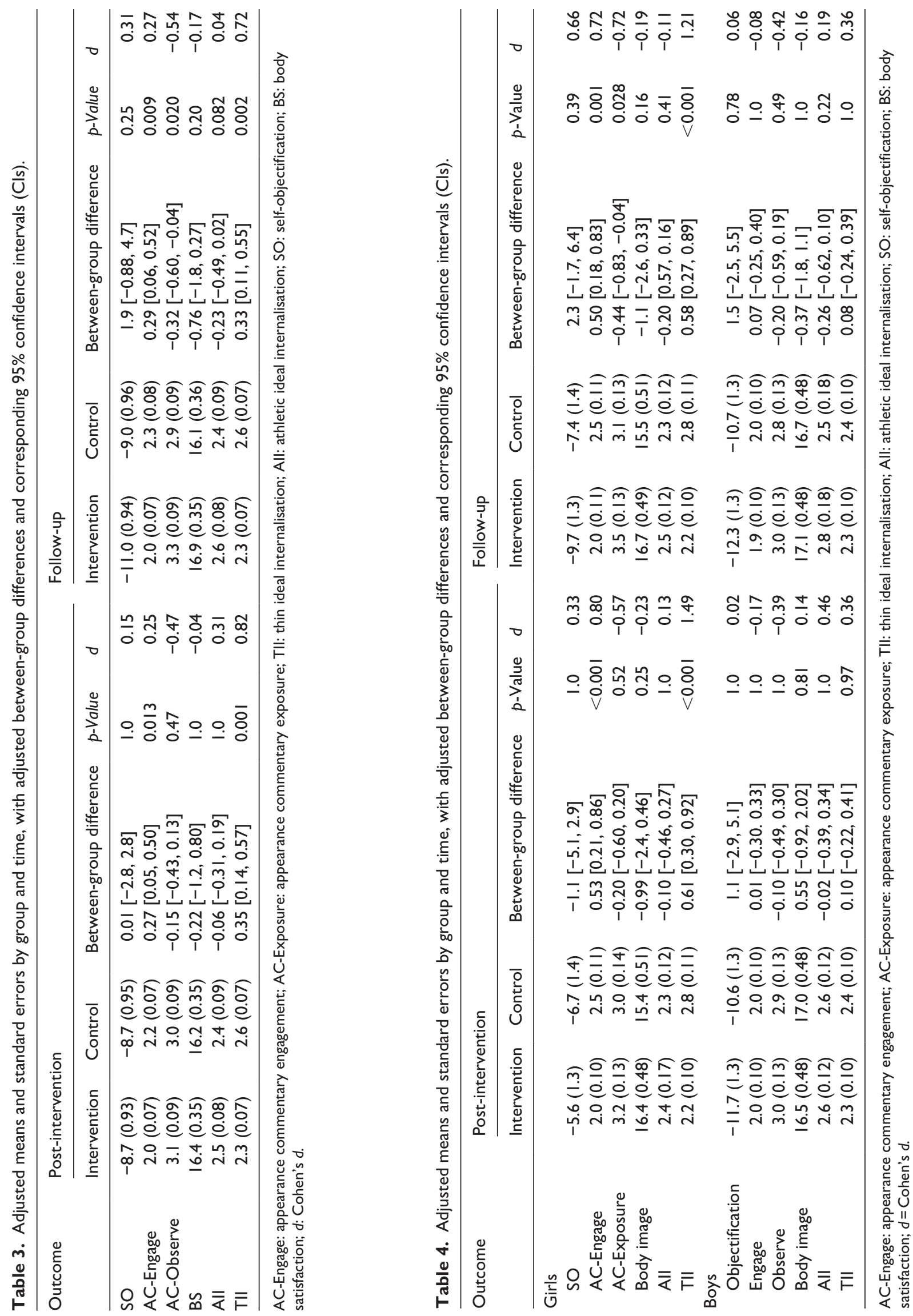


\section{Discussion}

The present study aimed to evaluate the efficacy of $B T I D A$; a classroom-based intervention that aims to reduce adolescents' engagement in appearance commentary and internalisation of sociocultural appearance messages, and increase body satisfaction. We found partial support for the efficacy of the intervention in achieving these aims. More specifically, girls who participated in $B T I D A$ reported significantly lower engagement in appearance commentary and thin ideal internalisation immediately post-intervention and at eight-week followup, than girls in the control group. They also reported greater exposure to appearance commentary at follow-up, perhaps reflecting an increased awareness of appearance commentary within the sociocultural environment that has grown over time. However, no intervention effects were found for other outcomes, or for boys.

$B T I D A$ offered a more targeted approach to challenging appearance commentary than existing classroom-based interventions, incorporating cognitive dissonance and critical literacy building activities that were explicitly focused on appearance commentary. In addition, we incorporated two additional behaviour change techniques (self-affirmation and implementation intentions) to bolster the intervention by facilitating reductions in appearance commentary outside of the intervention setting. This approach may explain why BTIDA was effective in creating lasting reductions in appearance commentary among girls, whereas other interventions were less effective (e.g. Dunstan et al., 2017; McLean et al., 2017). That said, BTIDA showed limited effectiveness in reducing girls' negative body image. Though girls who received the intervention reported lower thin-ideal internalisation, they did not report lower athletic ideal internalisation or self-objectification, or improved body satisfaction. Thus, developing girls' ability to critically engage with appearance commentary through the intervention, led them to perceive thinness related appearance ideals as less important to them, but didn't impact on other dimensions of body image as some existing interventions have (e.g. Diedrichs et al., 2015; McLean et al., 2017). It may be that simply reducing appearance commentary engagement is insufficient in improving girls' body image, and that activities present in previous more holistic interventions (but absent in ours) are needed to improve body image, such as restructuring body-related cognitions and/or critiquing media imagery (see Lewis-Smith et al., 2019 for a recent review).

However, BTIDA was ineffective for boys; no differences were found between the intervention and control group for boys in terms of appearance commentary engagement and body image. Consistent with past research (Bird et al., 2013; Diedrichs et al., 2015), boys reported lower baseline levels of body image and appearance commentary than girls. These low levels of key outcome variables at the start of the study, may have created a floor effect wherein any benefits of the intervention (e.g. in reinforcing current negative attitudes towards the target behaviour) were undetectable. Alternatively, it may mean that the issues addressed in the intervention were not relevant to, or not perceived as relevant to, boys - despite the efforts of our pilot work. Interventions that focus on more male-centric body image issues, such as drive for muscularity and anabolic steroid use, have been shown to foster positive body image outcomes for boys (Yager et al., 2019). Lastly, it is also possible that measurement may have been an issue, since the majority of our outcome measures were originally designed for use with female adolescents, and so may not have adequately captured male adolescents' body image risk and appearance concerns. Qualitative work is needed to understand the reasons underpinning boys' response to the intervention, with the aim of adapting the intervention content and/or evaluation procedures as appropriate.

\section{Implications}

In demonstrating the partial efficacy of BTIDA, we contribute to the wealth of existing research demonstrating the utility of cognitive dissonance and media literacy building techniques in reducing body image risk among adolescents (Lewis-Smith et al., 2019). In particular, we contribute to the evidence base demonstrating the flexibility of these techniques by using them to address a specific dimension of negative body image risk - in this instance, appearancerelated commentary. We also demonstrate the potential for augmenting such interventions with behaviour change techniques that have demonstrated efficacy in other health domains (namely, self-affirmation and implementation intentions), in order to maximise the efficacy of interventions in changing behaviours associated with negative body image. Moreover, BTIDA is a feasible intervention within school settings. It was initially designed in collaboration with a teacher as a psychologically informed lesson for teachers to deliver themselves in the classroom. The session lasts just sixty minutes and can easily be integrated into busy curriculums within secondary schools. Therefore, though in this study, the intervention was delivered to all classes by trained research assistants to minimise confounding variables, teacher-led delivery should be possible. That said, BTIDA was not as effective in reducing negative body image as other interventions, and so an alternative direction for future work would be to explore the possibility of integrating BTIDA into existing more comprehensive interventions for girls, in order to bolster their effects on appearance interactions.

\section{Limitations}

Just one school was involved in the present evaluation of BTIDA. While using the same school for both the 
intervention and control group ensures closely matched groups for comparison of intervention effects and is widely used in the initial evaluation of school-based initiatives (e.g. Bird et al., 2013; Halliwell et al., 2015; McLean et al., 2017), it does allow for contamination between groups. Thus, the effectiveness of the intervention may be underestimated. Future research should therefore use separate yet well-matched schools for intervention and control group. It should also make use of active control groups (e.g. who receive some form of body image education), as opposed to waiting list control, in order to help better identify and isolate the active mechanisms of the intervention. Lastly, the school involved in this research was a state-funded academy in a predominantly white suburban area, and so testing the programme with a more diverse ethnic, socioeconomic and geographic mix of adolescents is needed.

Measurement is also an issue. In particular, the measure of appearance commentary was created specifically for this study, due to the absence of suitable existing measures that capture both off- and on- line commentary. Though this measure was based on existing measures (Salk and EngelnMaddox, 2011) and demonstrated both content validity and internal reliability, more thorough validation is needed moving forwards. Timing of measurement might also have affected findings. Follow-up measures were taken eightweeks post intervention, as this fit best within the school time timetable, but past research has typically opted for more long-term evaluation (3-6 months). Therefore, more long-term evaluation is needed to better understand the sustainability of the intervention.

\section{Conclusions}

Our findings suggest that BTIDA can be an effective and feasible means of reducing appearance commentary and thin ideal internalisation in girls. However, more evaluation work, involving more diverse groups is needed to understand the extent to which these findings can generalised beyond the present sample. Future evaluation work should also aim to overcome the limitations in measurement that were present within this study. Furthermore, despite promising results for girls, BTIDA was not effective for boys. There are several possible reasons for this lack of efficacy, and so qualitative follow-up work is needed to better understand this.

\section{Declaration of conflicting interests}

The author(s) declared no potential conflicts of interest with respect to the research, authorship, and/or publication of this article.

\section{Funding}

The author(s) disclosed receipt of the following financial support for the research, authorship, and/or publication of this article: The research was funded by York St John University Impactful Research fund.

\section{ORCID iDs}

Beth T Bell iD https://orcid.org/0000-0002-6587-0336

Adam Bates (iD https://orcid.org/0000-0002-5481-2974

\section{References}

Allison PD (2012) Handling missing data by maximum likelihood. SAS Global Forum, April. SAS Institute Inc. Available at: http:/www.statisticalhorizons.com/wp-content/uploads/ MissingDataByML.pdf ( accessed 1 July 2020).

Be Real (2017) Somebody Like Me: A report investigating the impact of body image anxiety on young people in the UK, 17 January. Available at: https://www.berealcampaign.co.uk/ research/somebody-like-me ( accessed 1 July 2020).

Bell BT (2019) "You take fifty photos, delete forty nine and use one": A qualitative study of adolescent image-sharing practices on social media. International Journal of ChildComputer Interaction 20: 64-71.

Bird EL, Halliwell E, Diedrichs PC, et al. (2013) Happy Being Me in the UK: A controlled evaluation of a school-based body image intervention with pre-adolescent children. Body Image 10(3): 326-334.

boyd D (2014) It's Complicated: The Social Lives of Networked Teens. New Haven; London: Yale University Press.

Chen H and Jackson T (2012) Gender and age group differences in mass media and interpersonal influences on body dissatisfaction among Chinese adolescents. Sex Roles 66: 3-20.

Chua JYX, Tam W and Shorey S (2020) Research review: Effectiveness of universal eating disorder prevention interventions in improving body image among children: A systematic review and meta-analysis. Journal of Child Psychology and Psychiatry 61(5): 522-535.

Chua THH and Chang L (2016) Follow me and like my beautiful selfies: Singapore teenage girls' engagement in self-presentation and peer comparison on social media. Computers in Human Behavior 55: 190-197.

Cohen J (1988) Statistical Power Analysis for the Behavioural Sciences. New York, NY: Routledge Academic.

Cruz-Sáez S, Pascual A, Wlodarczyk A, et al. (2018) The effect of body dissatisfaction on disordered eating: The mediating role of self-esteem and negative affect in male and female adolescents. Journal of Health Psychology 25(8): 1098-1108.

Dakanalis A, Carrà G, Calogero R, et al. (2015) The developmental effects of media-ideal internalization and self-objectification processes on adolescents' negative body-feelings, dietary restraint, and binge eating. European Child \& Adolescent Psychiatry 24(8): 997-1010.

Deighton-Smith N and Bell BT (2018) Objectifying fitness: A content and thematic analysis of\# fitspiration images on social media. Psychology of Popular Media Culture 7(4): 467-483.

Diedrichs PC, Atkinson MJ, Steer RJ, et al. (2015) Effectiveness of a brief school-based body image intervention 'Dove Confident Me: Single Session' when delivered by teachers and researchers: Results from a cluster randomised controlled trial. Behaviour Research and Therapy 74: 94-104.

Döring N, Reif A and Poeschl S (2016) How gender-stereotypical are selfies? A content analysis and comparison with magazine adverts. Computers in Human Behavior 55: 955-962. 
Dunstan CJ, Paxton SJ and McLean SA (2017) An evaluation of a body image intervention in adolescent girls delivered in single-sex versus co-educational classroom settings. Eating Behaviors 25: 23-31.

Epton T, Harris PR, Kane R, et al. (2015) The impact of self-affirmation on health-behavior change: A meta-analysis. Health Psychology 34(3): 187-196.

Fredrickson BL and Roberts TA (1997) Objectification theory: Toward understanding women's lived experiences and mental health risks. Psychology of Women Quarterly 21(2): 173-206.

Frisén A and Berne S (2020) Swedish adolescents' experiences of cybervictimization and body-related concerns. Scandinavian Journal of Psychology 61(1): 68-76.

Gollwitzer PM and Sheeran P (2006) Implementation intentions and goal achievement: A meta-analysis of effects and processes. Advances in Experimental Social Psychology 38: 69-119.

Grogan S (2016) Body Image: Understanding Body Dissatisfaction in Men, Women and Children. London: Routledge.

Halliwell E, Jarman H, McNamara A, et al. (2015) Dissemination of evidence-based body image interventions: A pilot study into the effectiveness of using undergraduate students as interventionists in secondary schools. Body Image 14: 1-4.

Harris PR, Brearley I, Sheeran P, et al. (2014) Combining self-affirmation with implementation intentions to promote fruit and vegetable consumption. Health Psychology 33(7): 729-736.

Holmqvist Gattario K and Frisén A (2019) From negative to positive body image: Men's and women's journeys from early adolescence to emerging adulthood. Body Image 28: 53-65.

Jackson T and Chen H (2011) Risk factors for disordered eating during early and middle adolescence: Prospective evidence from Mainland Chinese boys and girls. Journal of Abnormal Psychology 120(2): 454-464.

Jones DC (2004) Body image among adolescent girls and boys: A longitudinal study. Developmental Psychology 40(5): $823-835$

Jones DC and Crawford JK (2006) The peer appearance culture during adolescence: Gender and body mass variations. Journal of Youth and Adolescence 35(2): 243.

Jones MD, Crowther JH and Ciesla JA (2014) A naturalistic study of fat talk and its behavioral and affective consequences. Body Image 11(4): 337-345.

Karazsia BT, Murnen SK and Tylka TL (2017) Is body dissatisfaction changing across time? A cross-temporal meta-analysis. Psychological Bulletin 143(3): 293-320.

Knauss C, Paxton SJ and Alsaker FD (2008) Body dissatisfaction in adolescent boys and girls: Objectified body consciousness, internalization of the media body ideal and perceived pressure from media. Sex Roles 59(9-10): 633-643.

Lawler M and Nixon E (2011) Body dissatisfaction among adolescent boys and girls: The effects of body mass, peer appearance culture and internalization of appearance ideals. Journal of Youth and Adolescence 40(1): 59-71.

Lewis-Smith H, Diedrichs PC and Halliwell E (2019) Cognitivebehavioral roots of body image therapy and prevention. Body Image 31: 309-320.

Livingstone S (2019) EU kids online. The International Encyclopedia of Media Literacy. Epub ahead of print 9 May 2019. DOI: 10.1002/9781118978238.ieml0065.
McLean SA, Wertheim EH, Masters J, et al. (2017) A pilot evaluation of a social media literacy intervention to reduce risk factors for eating disorders. International Journal of Eating Disorders 50(7): 847-851.

Neumark-Sztainer D, Paxton SJ, Hannan PJ, et al. (2006) Does body satisfaction matter? Five-year longitudinal associations between body satisfaction and health behaviors in adolescent females and males. Journal of Adolescent Health 39(2): 244-251.

Nichter M (2000) Fat Talk: What Girls and Their Parents Say About Dieting. Cambridge, MA: Harvard University Press.

Noll SM and Fredrickson BL (1998) A mediational model linking self-objectification, body shame, and disordered eating. Psychology of Women Quarterly 22(4): 623-636.

Ofcom (2020) Children and parents: Media use and attitudes report 2019. Ofcom, 1 November. Available at: https://www. ofcom.org.uk/_data/assets/pdf_file/0023/190616/childrenmedia-use-attitudes-2019-report.pdf

Puhl RM, Wall MM, Chen C, et al. (2017) Experiences of weight teasing in adolescence and weight-related outcomes in adulthood: A 15-year longitudinal study. Preventive Medicine 100: 173-179.

Richardson SM and Paxton SJ (2010) An evaluation of a body image intervention based on risk factors for body dissatisfaction: A controlled study with adolescent girls. International Journal of Eating Disorders 43(2): 112-122.

Rohde P, Stice E and Marti CN (2015) Development and predictive effects of eating disorder risk factors during adolescence: Implications for prevention efforts. International Journal of Eating Disorders 48(2): 187-198.

Salk RH and Engeln-Maddox R (2011) "If you're fat, then I'm humongous!" Frequency, content, and impact of fat talk among college women. Psychology of Women Quarterly 35(1): 18-28.

Schaefer LM, Burke NL, Thompson JK, et al. (2015) Development and validation of the sociocultural attitudes towards appearance questionnaire-4 (SATAQ-4). Psychological Assessment 27(1): 54-67.

Shannon A and Mills JS (2015) Correlates, causes, and consequences of fat talk: A review. Body Image 15: 158-172.

Shin T, Davison ML and Long JD (2017) Maximum likelihood versus multiple imputation for missing data in small longitudinal samples with non-normality. Psychological Methods 22(3): 426-449.

Slater A and Tiggemann M (2011) Gender differences in adolescent sport participation, teasing, self-objectification and body image concerns. Journal of Adolescence 34(3): 455-463.

Smolak L and Levine MP (2015) The Wiley Handbook of Eating Disorders. Chichester, UK: Wiley-Blackwell.

Tancred T, Fletcher A, Melendez-Torres GJ, et al. (2017) Integrating health education in academic lessons: Is this the future of health education in schools? The Journal of School Health 87(11): 807-810.

Thompson JK, Heinberg LJ, Altabe M, et al. (1999) Exacting Beauty: Theory, Assessment, and Treatment of Body Image Disturbance. Washington, DC: American Psychological Association.

Tiggemann M and Barbato I (2018) "You look great!": The effect of viewing appearance-related Instagram comments on women's body image. Body Image 27: 61-66. 
Trekels J, Ward LM and Eggermont S (2018) I "like" the way you look: How appearance-focused and overall Facebook use contribute to adolescents' self-sexualization. Computers in Human Behavior 81: 198-208.

Vandenbosch L and Eggermont S (2014) The three-step process of self-objectification: Potential implications for adolescents' body consciousness during sexual activity. Body Image 11(1): 77-80.

Wieber F, Thürmer JL and Gollwitzer PM (2015) Promoting the translation of intentions into action by implementation intentions: Behavioral effects and physiological correlates. Frontiers in Human Neuroscience 9: 395.

Wilksch SM, Paxton SJ, Byrne S, et al. (2015) Prevention across the spectrum: A randomized controlled trial of three programs to reduce risk factors for both eating disorders and obesity. Psychological Medicine 45(9): 1811-1823.
Yager Z, Diedrichs PC, Ricciardelli LA, et al. (2013) What works in secondary schools? A systematic review of classroombased body image programs. Body Image 10(3): 271-281.

Yager Z, McLean SA and Li X (2019) Body image outcomes in a replication of the ATLAS program in Australia. Psychology of Men \& Masculinity 20(3): 418-218.

Yamamiya Y, Shimai S, Schaefer LM, et al. (2016) Psychometric properties and validation of the sociocultural attitudes towards appearance questionnaire-4 (SATAQ-4) with a sample of Japanese adolescent girls. Body Image 19: 89-97.

Yamamiya Y, Shroff H, Schaefer LM, et al. (2019) An exploration of the psychometric properties of the SATAQ-4 among adolescent boys in Japan. Eating Behaviors 32: 31-36.

Yau JC and Reich SM (2018) Are the qualities of adolescents' offline friendships present in digital interactions? Adolescent Research Review 3(3): 339-355. 